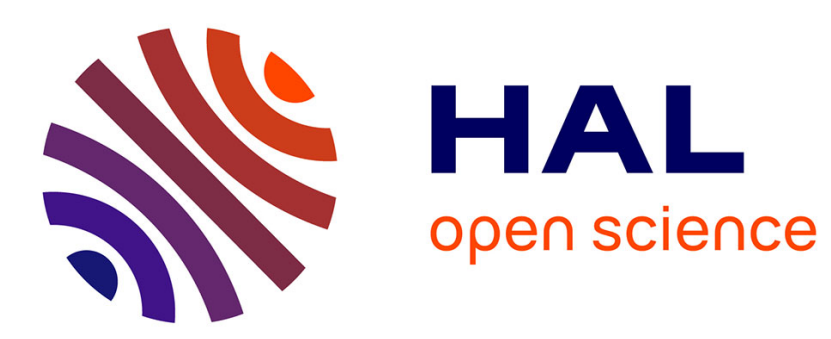

\title{
Numerical investigation of a three-dimensional four field model for collisionless magnetic reconnection
}

\author{
D. Grasso, D. Borgogno, Emanuele Tassi
}

\section{To cite this version:}

D. Grasso, D. Borgogno, Emanuele Tassi. Numerical investigation of a three-dimensional four field model for collisionless magnetic reconnection. Communications in Nonlinear Science and Numerical Simulation, 2012, 17, pp.2085. hal-00617464

\section{HAL Id: hal-00617464 \\ https://hal.science/hal-00617464}

Submitted on 29 Aug 2011

HAL is a multi-disciplinary open access archive for the deposit and dissemination of scientific research documents, whether they are published or not. The documents may come from teaching and research institutions in France or abroad, or from public or private research centers.
L'archive ouverte pluridisciplinaire HAL, est destinée au dépôt et à la diffusion de documents scientifiques de niveau recherche, publiés ou non, émanant des établissements d'enseignement et de recherche français ou étrangers, des laboratoires publics ou privés. 


\title{
Numerical investigation of a three-dimensional four field model for collisionless magnetic reconnection
}

\author{
D. Grasso ${ }^{1}$, D. Borgogno ${ }^{2}$, E. Tassi ${ }^{3}$ \\ 1. CNR Consiglio Nazionale delle Ricerche, Istituto dei Sistemi Complessi, Dipartimento \\ di Energetica, Politecnico di Torino Corso Duca degli Abruzzi 24, I-10129 Torino, Italy \\ 2. Dipartimento di Energetica, Politecnico di Torino, Corso Duca degli Abruzzi 24, 10129 \\ Torino, Italy \\ 3. Centre de Physique Théorique, CNRS - Aix-Marseille Universités, Campus de Luminy, \\ case 907, F-13288 Marseille cedex 09, France
}

\begin{abstract}
In this paper we present the numerical investigation of a three-dimensional four field model for magnetic reconnection in collisionless regimes. The model describes the evolution of the magnetic flux and vorticity together with the perturbations of the parallel magnetic and velocity fields. We explored the different behavior of vorticity and current density structures in low and high $\beta$ regimes, $\beta$ being the ratio between the plasma and magnetic pressure. A detailed analysis of the velocity field advecting the relevant physical quantities is presented. We show that, as the reconnection process evolves, velocity layers develop and become more and more localized. The shear of these layers increases with time ending up with the occurrence of secondary instabilities of the Kelvin-Helmholtz type. We also show how the $\beta$ parameter influences the different evolution of the current density structures, that preserve for longer time a laminar behavior at smaller $\beta$ values. A qualitative explanation of the structures formation on the different $z$-sections is also presented.
\end{abstract}

Key words: Nonlinear dynamics, plasma instabilities, fluid instabilities, numerical simulations

\section{Introduction}

Magnetic reconnection is a fundamental process in highly conductive fluids and plasmas $[1,2]$. It can be defined as a change in the topology of the magnetic field lines, which decouple their motion from the fluid one. It is 
associated with a release of magnetic energy into heat, plasma kinetic energy and fast particle energy and it is characterized by the formation of current density sheets in the reconnection region along with strong velocity layers. The range of phenomena involving magnetic reconnection is very wide. It includes solar flares, geomagnetic substorms, interaction of the solar wind with the magnetopause, sawtooth oscillations and disruptions in laboratory plasma, such as in Tokamaks. One of the problems in magnetic reconnection is to identify the appropriate generalized Ohm's law and the physical mechanisms which cause the diffusion of the magnetic field through the plasma. In weakly collisional plasmas, such as the high temperature ones in Tokamaks, the inverse of the electron-ion collision frequency is larger than the relaxation time of internal sawtooth oscillations. This consideration made collisionless reconnection to become a frontier subject in the early '90 [3, 4]. In this regime the typical length scale of the reconnection process is given by the collisionless skin depth, $d_{e}$. Although a kinetic approach should be invoked in order to treat such collisionless regimes, in the presence of a strong guide field, fluid models, which offer a computational advantage, are often used. Many studies have been done in the framework of two-fluid models. In this context a description of the plasma behavior can be made considering a simpler two-field description [5] or a more sophisticated four-field description [6]. In particular, the two-field model in [5] has been extensively analyzed in the last decade both in two-dimensional and three-dimensional configurations $[7,8,9,10]$. In this model the evolution of the magnetic flux and plasma stream function is followed assuming that variations of the magnetic field and of the plasma velocity, along the direction parallel to the guide field, are negligible. The fingerprint of this approach is the coupling between the evolution of the current density and vorticity fields, which evolve in ordered or turbulent structures depending on the value of the electron temperature, which enters the equation through the the ion sound Larmor radius $\varrho_{s}[7,9]$, which is a further characteristic scale length of the phenomenon. For values of $\varrho_{s} \geq d_{e}$ the velocity layers,advecting the current density and vorticity, evolve in ordered coherent structures aligned with the separatrix of the magnetic island. On the other hand, for $\varrho_{s}<<d_{e}$ the velocity layers tend to be aligned with the neutral line giving rise to strong shears that lead to the onset of secondary Kelvin-Helmholtz instabilities. This twofold picture has been also confirmed in three-dimensional configurations [10, 11]. When we allow the system to develop also magnetic and plasma velocity perturbations along the direction parallel to the guide field, by considering the four-field 
model in [6], the picture becomes richer and a new scenario appears, where the evolutions of the current density and vorticity fields are no longer coupled. The crucial role in this change is played by the $\beta$ parameter, expressing the ratio between the plasma and the magnetic field pressures. Indeed, when the low $\beta$ limit is abandoned, the effects of the strong velocity shears that develop in the reconnection region, on one hand lead to a turbulent vorticity, but on the other hand they get suppressed in the evolution of the current density $[11,12]$.

Recently, an extension of this four-field model to three dimensions has also been derived [13]. The model belongs to the class of fluid models for plasma physics, for which a noncanonical Hamiltonian formulation is known. The origin of this class can be traced back to the seminal work of Morrison and Greene [14] on ideal magnetohydrodynamics. The class was subsequently enlarged to a great extent by Phil Morrison and co-workers over the years, with the discovery of Hamiltonian structures for several reduced fluid models.

In this article we analyze this model by performing numerical simulations with the aim of investigating the evolution, and its dependence on $\beta$, of velocity and current density fields in a full 3D setting. In particular we address the question of understanding in what fields the secondary KelvinHelmholtz instability dominates and in what fields it is suppressed, and how such instability extends along the $z$ direction. We also recall that the absence of an ignorable coordinate raises the problem of interpreting a dynamics which is much less constrained than the two-dimensional one[15, 10, 11]. In particular, the system no longer possesses the infinity of Casimir invariants which constrain the $2 \mathrm{D}$ case and in terms of which it is possible to explain the different structures observed in the current density and vorticity fields $[5,8,16,12]$. Such explanation was indeed possible, due to the presence of advected scalar fields whose existence was related to the presence of an infinite number of Casimirs. The paper is organized as follows: in Sec. 2 we present the model equations; in Sec. 3 the simulation results are shown and discussed; in Sec. 4 we focus on the energy partition; conclusions are drawn in Sec. 5.

\section{Model equations}

Our investigation is based on the 3D four-field model for collisionless reconnection, described in Ref.[13]. Considering a Cartesian coordinate system $(x, y, z)$, with the constant magnetic guide field directed along $z$, the model 
equations are given by

$$
\begin{aligned}
\frac{\partial\left(\psi-d_{e}^{2} \nabla_{\perp}^{2} \psi\right)}{\partial t}+\left[\varphi, \psi-d_{e}^{2} \nabla_{\perp}^{2} \psi\right]-d_{\beta}[\psi, Z]+\frac{\partial \varphi}{\partial z}+d_{\beta} \frac{\partial Z}{\partial z} & =0 \\
\frac{\partial Z}{\partial t}+[\varphi, Z]-c_{\beta}[v, \psi]-d_{\beta}\left[\nabla_{\perp}^{2} \psi, \psi\right]-c_{\beta} \frac{\partial v}{\partial z}-d_{\beta} \frac{\partial \nabla_{\perp}^{2} \psi}{\partial z} & =0 \\
\frac{\partial \nabla_{\perp}^{2} \varphi}{\partial t}+\left[\varphi, \nabla_{\perp}^{2} \varphi\right]+\left[\nabla_{\perp}^{2} \psi, \psi\right]+\frac{\partial \nabla_{\perp}^{2} \psi}{\partial z} & =0 \\
\frac{\partial v}{\partial t}+[\varphi, v]-c_{\beta}[Z, \psi]-c_{\beta} \frac{\partial Z}{\partial z} & =0
\end{aligned}
$$

where $\psi$ is the poloidal magnetic flux function, $\varphi$ is the $\mathbf{E} \times \mathbf{B}$ stream function, $Z$ is proportional to the parallel magnetic perturbation and $v$ is the parallel plasma velocity. The parameter $d_{e}$ represents the electron skin depth, whereas the other two parameters $c_{\beta}$ and $d_{\beta}$ are defined by $c_{\beta} \equiv \sqrt{\beta /(1+\beta)}$, with $\beta$ indicating the ratio between the plasma pressure and the toroidal magnetic pressure, and by $d_{\beta} \equiv d_{i} c_{\beta}$, where $d_{i}$ is the ion skin depth. The Poisson bracket is defined by $[f, g]=\hat{z} \cdot(\nabla f \times \nabla g)$ for generic fields $f$ and $g$, whereas the symbol $\nabla_{\perp}$ refers to the gradient perpendicular to $\hat{z}$. Equations (1)-(4) are written in a dimensionless form, according to which, the time is normalized with respect to a characteristic Alfvèn time $t_{A}$, lengths are normalized with respect to a characteristic scale $L$ and magnetic fields with respect to a characteristic value $B$. If such value is taken to be the amplitude $B_{0}$ of the toroidal guide field, then the following ordering applies:

$$
\begin{aligned}
\psi \sim \varphi \sim Z \sim v \sim \frac{\partial}{\partial t} \sim \frac{\partial}{\partial z} \sim \epsilon & \equiv \frac{B_{p}}{B_{0}} \ll 1, \\
\frac{\partial}{\partial x} & \sim \frac{\partial}{\partial y} \sim 1,
\end{aligned}
$$

where $B_{p}$ indicates a characteristic value for the poloidal magnetic field (remark that this ordering differs from that of Ref. [13], in which a normalization based on $B_{p}$, instead of $B_{0}$, was adopted).

Note that the Eqs. (1)-(4) are exact only at the order $\epsilon^{2}$. Indeed, in their derivation, only the contributions up to order $\epsilon$ of the normalized magnetic field

$$
\mathbf{B}=\hat{z}+\nabla \psi \times \hat{z}+c_{\beta} Z \hat{z}+\mathcal{O}\left(\epsilon^{2}\right)
$$

have been used. A term $-c_{\beta} \int_{0}^{x} d x^{\prime} \partial Z / \partial z \hat{x}$, which is of order $\epsilon^{2}$, is required in order to have a divergence-free magnetic field, but it produces only higher 
order corrections to (1)-(4).

By construction, the system (1)-(4) is Hamiltonian and conserves the total energy

$$
H=\frac{1}{2} \int_{\mathcal{D}} d^{3} x\left(d_{e}^{2} J^{2}+\left|\nabla_{\perp} \varphi\right|^{2}+v^{2}+\left|\nabla_{\perp} \psi\right|^{2}+Z^{2}\right),
$$

where $J=-\nabla_{\perp}^{2} \psi$ is the parallel current density and $\mathcal{D}$ is the domain of interest. The corresponding Poisson bracket has been provided in Ref. [13].

\section{Numerical results}

The equations (1)-(4) are integrated numerically by splitting all the fields in two parts: an equilibrium, independent on time, and an evolving perturbation. The perturbed component is advanced in time by an explicit, fourth order Adam-Bashforth scheme. The equations are solved in a three dimensional slab, with periodic boundary conditions along the $y$ and $z$ directions. Dirichlet conditions are applied at the edges of the $x$ axis, imposing that all the perturbed fields go to zero. A compact finite difference [17] algorithm, suitable for non-equispaced grid, is used for the spatial operations along the $x$ direction, while pseudo-spectral methods are adopted for the periodic directions. Numerical filters are introduced in order to control the numerical error propagation, which is a relevant issue in the absence of any dissipative effect, as in the problem we are considering. As described in [17], these filters smooth out the small spatial scales below a chosen cutoff, while leaving unchanged the large scale dynamics on all the time evolution of the process. Finally, in order to address the three-dimensional problem, the code has been parallelized adopting the MPI libraries. We set up a numerical experiment of spontaneous, 3D, collisionless reconnection process in a static equilibrium configuration with

$$
\psi_{e q}=-\log \cosh (x) \text { and } \varphi_{e q}=v_{e q}=Z_{e q}=0 .
$$

The integration domain is defined by $-L_{x}<x<L_{x},-L_{y}<y<L_{y}$ and $-L_{z}<z<L_{z}$, where $L_{x}=11.32, L_{y}=4 \pi, L_{z}=64 \pi$. The choice of $L_{x}$ avoids any influence of boundary conditions imposed along the $x$ direction on the reconnection dynamics. The value of $L_{y}$ allow to consider the magnetic reconnection instability induced by highly unstable modes, that in the twodimensional limit fall in the so-called large $\Delta^{\prime}$ regime. It is accepted $[3,4,18]$ that in this regime magnetic reconnection can develop on a relatively fast 
time scale. Finally, the choice of $L_{z}>>L_{y}$ guarantees that $k_{z}=2 \pi n / L_{z}<$ $k_{y}=2 \pi m / L_{y}$ for all the pairs of mode numbers $(m, n)$ that develop during the nonlinear evolution of the process, as imposed by the conditions (5)-(6). In order to take into account the $3 \mathrm{D}$ effects on the magnetic reconnection we perturbed the equilibrium (9) by introducing the following double helicity perturbation on the current density field

$$
\delta J(x, y, z)=\hat{J}_{1}(x) \exp \left(i k_{y 1} y+i k_{z 1} z\right)+\hat{J}_{2}(x) \exp \left(i k_{y 2} y-i k_{z 2} z\right) .
$$

Here we considered the pairs of wave numbers $(1,1)$ and $(1,-1)$, that correspond to the wave vectors components $k_{y 1}=1 / 4, k_{z 1}=1 / 64$ and $k_{y 2}=1 / 4$, $k_{z 2}=-1 / 64$. These two helicities, that are both linearly unstable, have resonant surfaces at $x_{s 1}=0.06258$ and $x_{s 2}=-0.06258 . \hat{J}_{1}(x)$ and $\hat{J}_{2}(x)$ are functions localized within a width of the order $d_{e}$, around $x_{s 1}$ and $x_{s 2}$, respectively. Their typical amplitude is of order $10^{-4}$. In order to properly treat the small scale structures that typically generate in the collisionless regimes a mesh of $n x=801, n y=n z=512$ grid points has been adopted. In this paper we analyzed the influence of the 3D effects on the dynamics of the magnetic reconnection instability by considering two sets of the physical parameters appearing in eqs. (1)-(4). In both cases we have used $d_{e}=0.24$ and $d_{\beta}=0.96$, while $c_{\beta}$ is varied, assuming the values $c_{\beta}=0.4$ and $c_{\beta}=0.8$. This choice allows to address two regimes with different values of the ion skin depth $d_{i}$ and of the ratio, $\beta$, between the plasma and the magnetic field pressure. In particular, when $c_{\beta}=0.4, \beta=0.19$, while $c_{\beta}=0.8$ gives $\beta=1.78$, which we refer to as a small and a high $\beta$ regimes.

\subsection{Qualitative aspects of the dynamics along the $z$ direction}

As shown in the framework of a 3D, two-field, dissipationless model ([15],[10], [11]), the nonlinear interaction between two initially imposed linearly unstable helicities strongly affects the spatial distribution of the small scale structures where current density and vorticity fields are located. The shape of these patterns is the consequence of the interaction between the current layers associated to the island chains corresponding to the imposed helicities. According to the $2 \mathrm{D}$ results [7], in the collisionless cases when $\varrho_{s} \neq 0$ the current density field exhibits a cross-shape structure distributed along the separatrices of the magnetic islands, and is strongly peaked at the corresponding $\mathrm{X}$ points. For a single helicity with $\left(k_{y}, k_{z}\right)$ wave vector, the value of the $y$ coordinate of the magnetic island chain $\mathrm{X}$ points, varies along 
$z$ following the lines with slope $k_{z} / k_{y}$, while the value of the $x$ coordinate does not depend on $z$. This remains valid also in presence of more than one helicity, at least at the very early stages of the nonlinear evolution. In the early phase, indeed, each helicity is characterized by a given number, depending on $k_{y}$, of thin current density channels with a characteristic spatial orientation. Similarly to electric wires, the current density channels of various helicities interact among each other by means of attracting forces whose strength depends on their geometry. In particular, the maximum of the attraction is localized on the $z=$ const sections where the centers of the current density channels, localized at the X-points of the corresponding magnetic islands, have the same $y$ coordinates. On these sections the interaction leads to the merging of the current channels. An opposite scenario characterizes the $z=$ const planes where the $\mathrm{X}$ and O-points of the magnetic islands with different helicities face each other. Due to the fact that negative minimum current density values lie on the $\mathrm{O}$ points, very small attracting or even repulsive interactions act on the maximum current density peaks, as it is the case when two electric wires with currents flowing in opposite directions, face each other. Fig. 1 sketches this behavior for a double helicity case with poloidal and toroidal mode number $m=1, n=1$ and $m=1, n=-1$. The figure shows the current density distribution on four $z=$ const sections at the times $t_{1}$ and $t_{2}$, that correspond to the very beginning and the advanced phases of the nonlinear evolution of the process. In the planes $z=-L_{z}$ and $z=0$, where the $\mathrm{X}$ points of the structures have the same $y$ coordinate, the current layers experience the maximum attraction and merge by generating a single $\mathrm{X}$ point pattern at $t=t_{2}$. On such planes, the dynamics is quite similar to that observed in the single-helicity case. On the contrary, on the planes $z=L_{z} / 2$ and $z=-L_{z} / 2$, where the maximum current density of an island chain faces the minimum of the second chain, current layers merge by generating a new spatial distribution where two X-points are still present. The above described situations refer to the two extreme cases that can take place. Through the $z=$ const planes, lying between those described above, the dynamics varies continuously with $z$, with a force between the

current layers, which depends on the relative shift between the positions of the X-points along the $y$ direction.

\subsection{Interpretation of the numerical simulations}

Due to the high value of $d_{\beta}$, the early stages of the nonlinear evolution of the reconnection process are similar in both the cases $c_{\beta}=0.4$ and $c_{\beta}=0.8$. 
We define here the end of the linear phase by the time in which the growth of the two initially imposed modes, deviates from the exponential one, which they would have when considered separately. In this early nonlinear phase of the evolution, the contribution of the terms proportional to $c_{\beta}$ is almost negligible with respect to the Poisson brackets involving $d_{\beta}$. Since, in this limit, the parallel magnetic field perturbation $Z$ becomes proportional to the vorticity $U$, the equations for $F=\psi-d_{e}^{2} \nabla_{\perp}^{2} \psi$ and for $U$ decouple from the equations for $Z$ and $v$ and reduce to the two field limit of the model (1)(4), described in [15], where $\rho_{s}=d_{\beta}$ [16]. According to the two field model results [11], also in this case the current density and vorticity field concentrate in analogous structures characterized by thin and sharp layers on all the $z=$ const sections. Due to the proportionality with $U$, similar patterns develop also in $Z$, while the parallel velocity component $v$ is characterized by large scale cell structures similarly to $v_{\perp}=\left|\nabla_{\perp} \varphi\right|$, the field which it is mainly transported by. This behavior is illustrated in figure 2 , where the contour plots of $U, Z, J$ and $v$ are plotted on the particular plane $z=0$ at $t=90 \tau_{A}$ for the $c_{\beta}=0.8$ case.

When the influence of $c_{\beta}$ effects becomes important, at the later stages of the process, the vorticity layers tend to broaden, starting from the $z=$ const sections where the $\mathrm{X}$ points of the linear magnetic islands associated with the different helicities have similar $y$ coordinate. This leads to the formation of new vorticity structures, localized around the $\mathrm{x}=0$ axis for the particular choice of symmetric initial conditions we assumed, and, as a consequence, of highly localized, bar shaped, perpendicular velocity layers similar to jets as shown in figure 3. As already pointed out in Ref. [13], when the influence of a finite $\beta$ is not negligible, the structures of $U$ and $Z$ eventually differ. On the other hand, from Eqs. (2) and (3), one sees that in the limit $c_{\beta} \rightarrow 0$ (but with finite $d_{\beta}$ ) the relation $Z=d_{\beta} U$ satisfies the system, if the initial conditions on $Z$ and $U$ are the same, which is the case here. This explains the similarity in the structures of $Z$ and $U$ for negligible $\beta$.

The amplitude of the perpendicular velocity tends to become stronger as the magnetic reconnection develops. In the advanced nonlinear phase it provides the dominant contribution both in the equations of the vorticity and the parallel velocity fields. $U$ and $v$ are simply transported along the perpendicular velocity and as a consequence they assume the same spatial distribution of $v_{\perp}$. On the other hand, due to the value of $d_{\beta}$ we considered, the time evolution of $J$ and $Z$ continues to be dominated by the nonlinear terms involving the perpendicular component of the magnetic field, $d_{\beta}[\psi, Z]$ and $d_{\beta}\left[\nabla_{\perp}^{2} \psi, \psi\right]$ 
respectively. In figure 4 the intense jets appearing on the $z=0$ section for the perpendicular and parallel velocities are shown together with the more ordered structures characterizing $Z$ and $J$.

The fronts of both the parallel and perpendicular velocity jets tend to move towards the center of the computational box starting from the boundary surfaces $y= \pm 4 \pi$. Approaching the $y=0$ plane, the tops of the jets roll up and assume a "mushroom cap" shape which makes the velocity layers very similar to the "finger" like structures typical of the hydrodynamic RayleighTaylor instability. This suggests the fluid like behavior of the system in these structures. Despite the magnetic field line stochasticity which characterizes the $3 D$ settings we treat here, in fact, magnetic field components parallel to the jets are practically zero, which makes the contribution of the Poisson brackets $\left[\nabla_{\perp}^{2} \psi, \psi\right]$ and $[Z, \psi]$ locally negligible and reduces the $U$ and $v$ equations to purely fluid advection equations. This justifies the occurrence, at later times, when the width of the jets is of the order of $d_{e}$ and the velocity is comparable to $v_{A}$, of a Kelvin-Helmholtz like secondary instability which is responsible for the break of the velocity patterns.

At this stage $c_{\beta}$ effects appear also on $J$ and $Z$ fields producing a turbulent redistribution of the patterns in the regions where $v_{\perp}$ and $v$ are picked, which coexists with the laminar filaments concentrated at the boundaries of the reconnection area. This behavior is clearly illustrated in fig. 5, where the perpendicular velocity and current density are shown on the $z=8 \pi$ section at the latest stage of the process for the case $c_{\beta}=0.8$.

On the $x-y$ planes with a larger distance between the $y$ coordinates of the linear magnetic island X-points the contribution of $c_{\beta}$ remains rather small all along the reconnection process. On these sections, in fact, the nonlinear coupling between the current density structures do not allow the formation of the intense and extremely localized velocity layers described above, as illustrated in fig. 6. Here a comparison of the merging of the current density layers is shown on two different planes. On the left is the contour plot as it appears at $z=-64 \pi$ and on the right as it appears at $z=32 \pi$ plane. These planes correspond to the first and second sketch of fig. 1 respectively. We can see that the current density layers tend to broaden and their intensity is reduced as far as we consider $z$-planes where the distance between the $y$ coordinates of the $X$-points, corresponding to the two initially imposed helicities, increases. Because of the dominant terms depending on $d_{\beta}$, the $U, J$ and $Z$ fields exhibit analogous patterns, while $v_{\perp}$ and $v$ are characterized by spatial structures larger than $d_{e}$ and amplitude much smaller than $v_{A}$. 
It is also interesting to comment on the difference between a small and a high $\beta$ regimes, which we consider as represented by the $c_{\beta}=0.4$ and $c_{\beta}=0.8$ cases, respectively. In particular, in the high- $\beta$ regime, one has $d_{\beta} \approx d_{i}$ and this parameter measures the strength of the Hall term in the equations governing the magnetic dynamics. In the $2 \mathrm{D}$ limit, the model becomes then identical to the Hall MHD model investigated in Ref. [19], apart from the difference in the mechanism breaking the frozen-in condition. The comparison between the $c_{\beta}=0.4$ and $c_{\beta}=0.8$ cases confirms that also in $3 D$ geometry larger values of the $\beta$ parameter accelerate the formation of the fluid jets, whose occurrence happens at earlier stages of the reconnection process. Parallel and perpendicular velocities inside the layers are typically smaller in the lower $\beta$ case, for comparable extensions of the reconnection region on a given $z=$ const section. This difference is highlighted in fig. 7 , where the profiles of the velocity fields $v_{\perp}$ and $v$ are shown on the section $z=0$ for the two different values of $c_{\beta}$ mentioned above. In particular, on the first row $v_{\perp}$ (left frame) and $v$ (right frame) are plotted as function of $x$, in the limited range $-4<x<4$ and $-2<x<2$ respectively, at the $y$ coordinate corresponding to their maximum. We see that, when considered at times which correspond to the same phase of the evolution of the reconnection process in the two cases, the velocity fields are smaller by a factor of order two in the lower $c_{\beta}$ limit, drawn in red. This characteristic is seen also in the second row of fig. 7, where the velocity fields are plotted as function of $y$ at $x=0$, where the fluids jets are located. Here, we can also appreciate that the extension of the fluid jets, which start at the boundaries $y= \pm 4 \pi$ for both cases, is shorter when $c_{\beta}=0.4$. In this case (red lines) the jets end at $y= \pm 5$ while for $c_{\beta}=0.8$ (black lines) they end at $y= \pm 1$ approximately. This is due to the fact that the jets formation started earlier for the $c_{\beta}=0.8$. As it will be seen in the next section, greater outflow speeds in the parallel direction, at high $\beta$, can be ascribed to a transfer of energy, mediated by the Lorentz force, from the magnetic to the kinetic form. The amount of transferred energy is indeed proportional to $c_{\beta}$. Therefore one can expect a greater acceleration at higher $\beta$. We remark also that, because the Hamiltonian (8) is independent on $\beta$, the total energy available in the small and high $\beta$ case is the same. Therefore, increase of the parallel kinetic energy with $\beta$ is a pure consequence of the dynamical transfer and is not influenced by differences in the total energy of the system. Finally, we notice that, as a consequence of having less steep velocity layers for small $\beta$, in the latter case the KelvinHelmholtz destabilization of the fluid jets is delayed, making its influence on 
the density current and parallel magnetic field perturbation rather smooth.

\section{Energy considerations}

Because the model under consideration is a Hamiltonian system, the total energy (8) should be preserved during the dynamics. It is therefore important to verify that the decay of $H$, due to numerical dissipation, remains negligible. From Fig. 8 it is possible to see that, at the latest time of the simulation, the drop in the total energy for both the cases reported is less than $4 \%$. We remark also that, during most of the simulations, the velocity at which the magnetic energy decreases is much larger than that at which the total energy dissipates. This reassures us of the fact that the magnetic energy loss is due to a genuine reconnection (or also ideal) process and not to numerical dissipation.

In addition to remarks concerning the reliability of the simulations, the analysis of the time evolution of the different forms of energy is also suitable for physical considerations. Fig. 8 shows how, evidently, the perpendicular magnetic energy is converted into various forms during the reconnection process. In order to better understand the mechanisms of transfer of energy, it is useful to consider the following relations, which can be easily obtained from the model equations (1)-(4):

$$
\begin{aligned}
\frac{d}{d t} \frac{1}{2} \int d^{3} x\left|\nabla_{\perp} \varphi\right|^{2} & =\int d^{3} x J(\mathbf{B} \cdot \nabla) \varphi+\mathcal{O}\left(\epsilon^{4}\right), \\
\frac{d}{d t} \frac{1}{2} \int d^{3} x v^{2} & =c_{\beta} \int d^{3} x v(\mathbf{B} \cdot \nabla) Z+\mathcal{O}\left(\epsilon^{4}\right), \\
\frac{d}{d t} \frac{1}{2} \int d^{3} x\left(\left|\nabla_{\perp} \psi\right|^{2}+d_{e}^{2} J^{2}\right) & =-\int d^{3} x J(\mathbf{B} \cdot \nabla) \varphi \\
-d_{\beta} \int d^{3} x J(\mathbf{B} \cdot \nabla) Z+\mathcal{O}\left(\epsilon^{4}\right), & \\
\frac{d}{d t} \frac{1}{2} \int d^{3} x Z^{2} & =-c_{\beta} \int d^{3} x v(\mathbf{B} \cdot \nabla) Z \\
+d_{\beta} \int d^{3} x J(\mathbf{B} \cdot \nabla) Z+\mathcal{O}\left(\epsilon^{4}\right) . &
\end{aligned}
$$

In order to obtain compact and physically more meaningful expressions, we express the right-hand sides of (11)-(14) in terms of the magnetic field B, instead of $\psi$ and $Z$. As a consequence, by virtue of what was specified 
in Sec.2, the resulting expressions are exact only at the leading order and corrections of order $\epsilon^{4}$ are required. Of course, giving up $\mathbf{B}$ in favor of $\psi$ and $Z$, yields the relations whose sum gives the exact conservation of the Hamiltonian $H$. Note also that all the leading order terms on the right-hand sides of $(11)-(14)$ possess the common form $\int d^{3} x f(\mathbf{B} \cdot \nabla) g$, for generic fields $f$ and $g$. Mechanisms that produce the transfer of energy from one form to another, can then become inefficient if some fields have a weak variation along the magnetic field lines.

From Eqs. (11) and (13), we see that the mechanism that acts as a source for perpendicular kinetic energy, is a sink for the energy of the poloidal magnetic field and of the parallel current. The rise of $E_{k p e r p}$ observed in Fig. 8, then implies a drop in $E_{B}+E_{k e}$. The growth of the parallel kinetic energy, on the other hand, is possible only at finite $\beta$, in which case the parallel Lorentz accelerates the fluid. This mechanism acts also as a sink for the parallel magnetic energy. Note that, in Fig. 8, for the smaller $c_{\beta}$ case, there is a competition between the conversion of magnetic energy into parallel magnetic energy and perpendicular kinetic energy, which lasts until around $t=125$ i.e. well into the nonlinear phase, when $E_{Z}$ reaches its maximum and then drops. At the same time, an increase in $E_{v}$ is observed. On the other hand, for the larger $c_{\beta}$ case, the conversion into perpendicular plasma kinetic energy, is dominant from the beginning of the nonlinear phase. We notice also that the form of energies that grow the most in the last phase of the simulations, i.e. $E_{v}$ and $E_{k p e r p}$, are those determined by the fields, $v$ and $\left|\nabla_{\perp} \varphi\right|$, respectively, which exhibit the secondary instability.

\section{Conclusions}

In this paper we analyzed a three-dimensional model for collisionless reconnection, which takes into account magnetic and velocity perturbations along the guide field direction. On the basis of previous two-dimensional studies we were interested in exploring in a 3D context the different behavior of the current density and vorticity structures. To this aim, we carried out a detailed analysis of the plasma velocity fields, which play a key role in the current density and vorticity dynamics. In the presence of high values of the $d_{\beta}$ parameter, we found that, during the early stages of the nonlinear phase, the perpendicular and parallel velocities on the $z=$ const sections are characterized by large scale cell structures. The other fields $J, U$ and $Z$, on the other hand, develop thinner spatial patterns similar to the case 
$\beta=0$. Following the evolution process, we observe that the perpendicular velocity tends to form highly localized patterns, aligned along the $x=0$ line, on some peculiar $z=$ const sections, which depend on the particular choice of the initial perturbation. These patterns are then recaptured also in the parallel velocity and vorticity fields, while, due to the high value of the $d_{\beta}$ parameter chosen for this analysis, the evolution of the current density and of the $Z$ field, is dominated by the contribution of the Poisson brackets involving the magnetic flux.

At the end of the reconnection process both the perpendicular and parallel velocity layers have become so localized and intense that they develop secondary instabilities of the Kelvin-Helmholtz type. In connection with this we find that the larger $c_{\beta}$, the sooner these instabilities develop.

The increase of the velocities fields and the occurrence of secondary instabilities influence also the energy distribution, favoring the conversion of the perpendicular magnetic energy into perpendicular and parallel kinetic energy rather than into parallel magnetic energy. The latter, in particular is transferred into parallel kinetic energy through the action of the parallel Lorentz force.

\section{Acknowledgments}

This article is dedicated to P.J. Morrison, on the occasion of his 60th birthday. The authors thank Phil for many inspiring discussions had over the years. The authors acknowledge dr. L. Comisso for providing the sketch proposed in figure 1. E. T. acknowledges fruitful discussions with the Nonlinear Dynamics group at the Centre de Physique Théorique, Luminy. This work was supported by the European Community under the contracts of Association between EURATOM and ENEA and between EURATOM, CEA, and the French Research Federation for fusion studies. The views and opinions expressed herein do not necessarily reflect those of the European Commis-

sion. Financial support was also received from the Agence Nationale de la Recherche (ANR GYPSI).

\section{References}

[1] E.R. Priest and T.G. Forbes, Magnetic Reconnection, (Cambridge: Cambridge University Press) (2000). 
[2] D. Biskamp, Magnetic Reconnection in Plasmas, (Cambridge: Cambridge University Press) (2000).

[3] A.Y. Aydemir, Phys. Fluids, B4, 3469 (1992).

[4] M. Ottaviani, and F. Porcelli, Phys. Rev. Lett., 71, 382 (1993).

[5] T.J. Schep, F. Pegoraro and B.N. Kuvshinov, Phys. Plasmas, 1, 2843 (1994).

[6] R. Fitzpatrick and F. Porcelli, Phys. Plasmas 11, 4713 (2004); Phys. Plasmas 14, 049902 (erratum) (2007).

[7] E. Cafaro, D. Grasso, F. Pegoraro, F. Porcelli, and A. Saluzzi, Phys. Rev. Lett., 80, 4430 (1998).

[8] D. Grasso, F. Califano, F. Pegoraro and F. Porcelli, Phys. Rev. Lett., 86,5051 (2001).

[9] D. Del Sarto, F. Califano and F. and Pegoraro, Phys. Rev. Lett., 91, 235001-1 (2003).

[10] D. Grasso, D. Borgogno and F. Pegoraro, Phys. Plasmas, 14, 055703-1 (2007).

[11] D. Grasso, D. Borgogno, F. Pegoraro and E. Tassi Nonlin. Processes Geophys., 16, 241, (2009).

[12] E. Tassi, D. Grasso, F. Pegoraro, Commun. Nonlinear Sci. Numer. Simulat, 15, 2, (2010).

[13] E. Tassi, P.J. Morrison, D. Grasso, F. Pegoraro, Nucl. Fusion 50, 034007 (2010).

[14] P.J. Morrison, J.M. Greene, Phys. Rev. Lett. 45, 790 (1980)

[15] D. Borgogno, D. Grasso, F. Califano, F. Farina, F. Pegoraro, F. Porcelli,Phys. Plasmas., 12, 032309 (2005).

[16] E. Tassi, P.J. Morrison, F.L. Waelbroeck and D. Grasso, Plasma Phys. Control. Fusion 50, 085014 (2008).

[17] S.K. Lele, J. Comp. Phys. 103, 16 (1992). 
[18] R.G. Kleva, J.F. Drake and F.L. Waelbroeck, Phys. Plasmas 2, 23 (1995); X. Wang and A. Bhattacharjee, Phys. Rev. Lett. 70, 1627 (1993).

[19] L.F. Morales, S. Dasso, D.O. Gómez and P.D. Mininni, Adv. in Space Research, 37, 1287 (2006). 


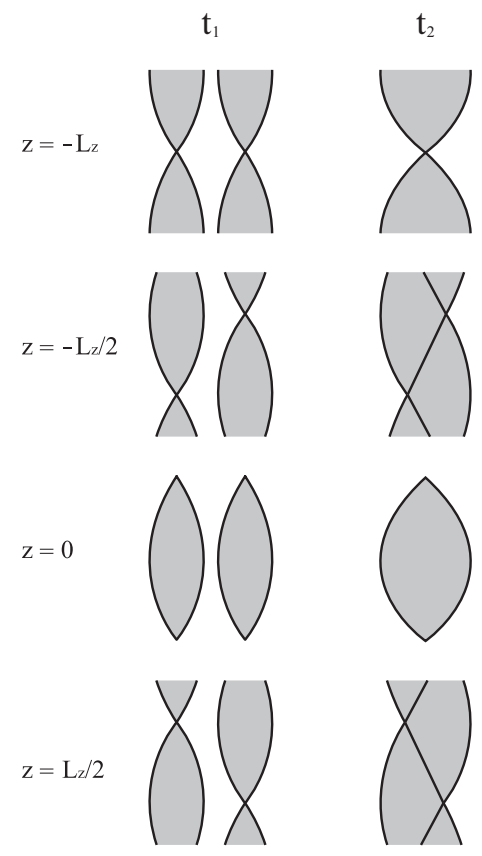

Figure 1: Sketch of the dynamics of the current density structures (black solid lines) in the nonlinear evolution of the $3 D$ reconnection process. The two columns shows the characteristic contour plots of the current density at an early, $t_{1}$, and at a more advanced stage, $t_{2}$, of the nonlinear phase on four different $z=$ const sections. In each plot the gray regions represent the corresponding domains where the magnetic topology modifications produced by the reconnection instability are located. 

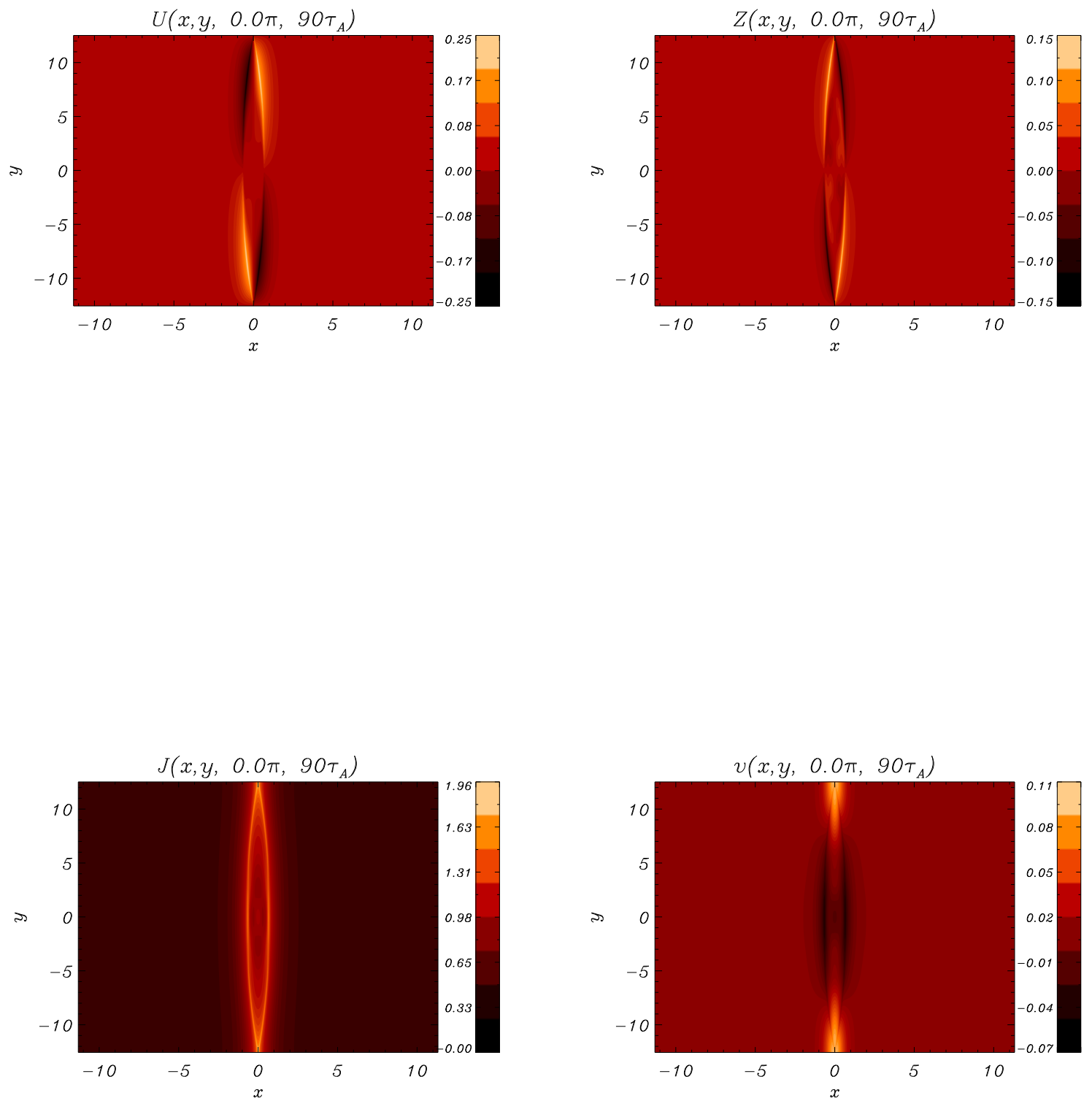

Figure 2: Contour plots of the vorticity $U$ (left top frame), the parallel magnetic field perturbation $Z$ (right top frame), the current density $J$ (left bottom frame) and the parallel velocity $v$ (right bottom frame) on the section $z=0$ at the time $t=90 \tau_{A}$, corresponding to the early nonlinear evolution, for the $c_{\beta}=0.8$ case. 

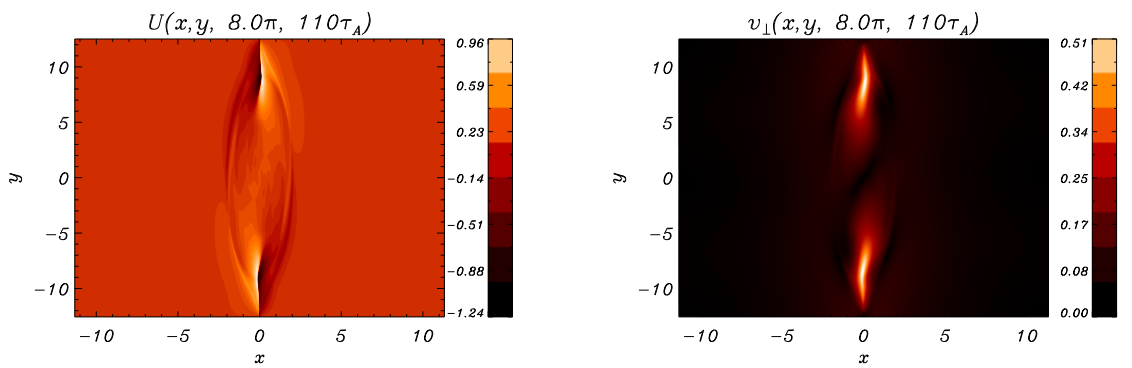

Figure 3: Contour plots of the vorticity $U$ (left frame) and the parallel velocity $v$ (right frame) on the section $z=8 \pi$ at the time $t=105 \tau_{A}$, for the $c_{\beta}=0.8$ case. Early fluid jets are located around the $x=0$ line. 

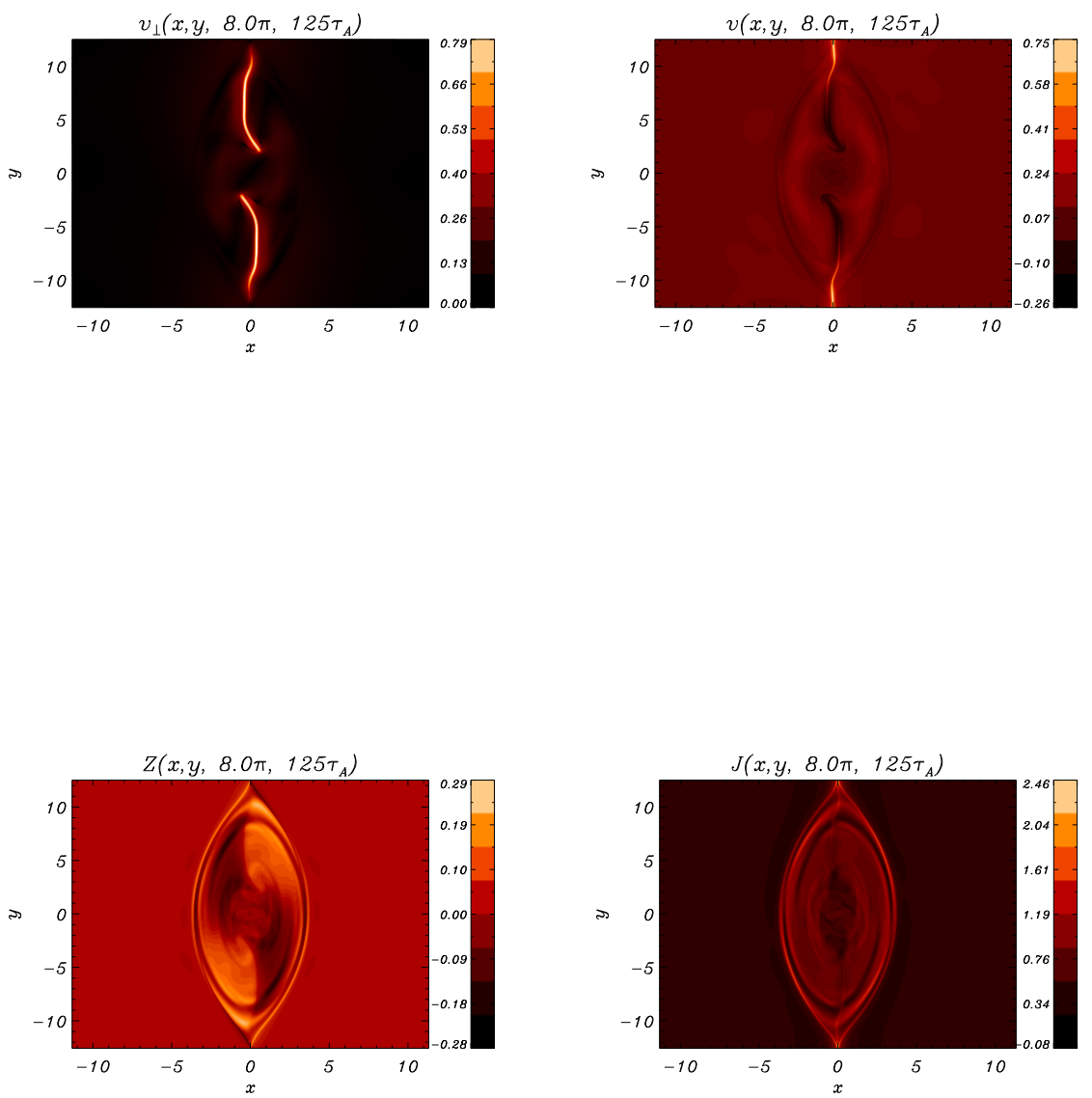

Figure 4: Contour plots of the perpendicular velocity $v_{\perp}$ (left top frame), the parallel velocity $v$ (right top frame), the parallel magnetic field perturbation $Z$ (left bottom frame) and the current density $J$ (right bottom frame) on the section $z=8 \pi$ at the time $t=125 \tau_{A}$, for the $c_{\beta}=0.8$ case. Jet like patterns are present in both the velocity components, whose maximum amplitude is of the order of the Alfvèn velocity, while $Z$ and $J$ exhibit a filamentary structure distributed on a large fraction of the corresponding area where the magnetic topology modifications are located. 

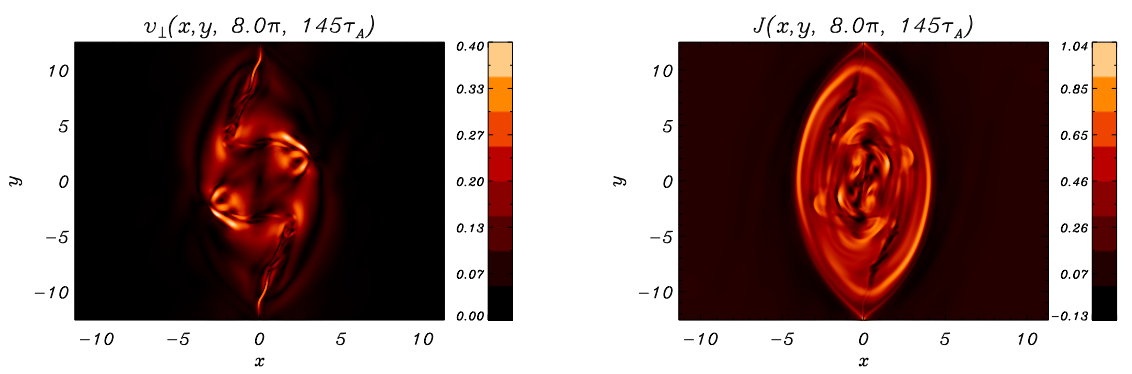

Figure 5: Contour plots of the pependicular velocity $v_{\perp}$ (left frame) and the current density $J$ (right frame) on the section $z=8 \pi$ at the time $t=145 \tau_{A}$, for the $c_{\beta}=0.8$ case. The occurrence of a secondary Kelvin-Helmholtz instability is responsible for the break of the $v_{\perp}$ field layers. At this stage of the process, a turbulent redistribution also affects the $J$ structures, especially along the layers where $v_{\perp}$ is concentrated. 

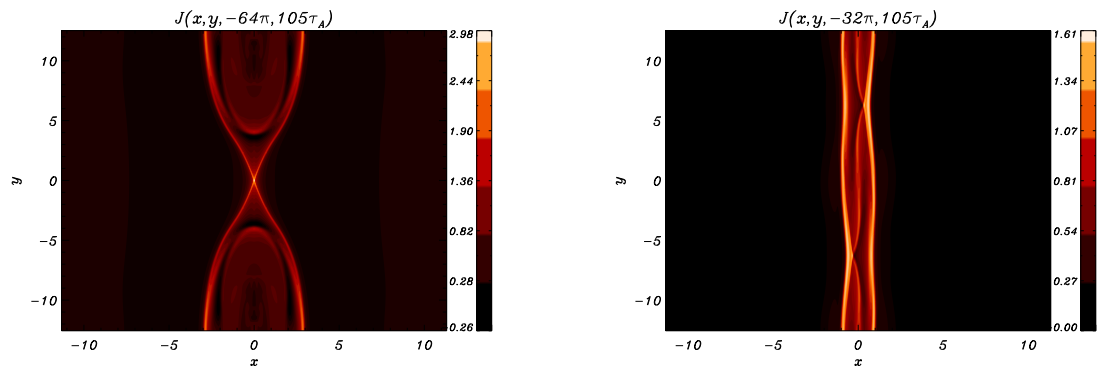

Figure 6: Contour plots of the current density on the $z=-64 \pi$ (left frame) and on the $z=-32 \pi$ (right frame) at the time $t=105 \tau_{A}$, for the $c_{\beta}=0.4$ case. The current density layers tend to broaden and their intensity is reduced as far as we consider $z$-planes where the distance between the $y$ coordinates of the $X$-points, corresponding to the two initially imposed helicities, increases. 

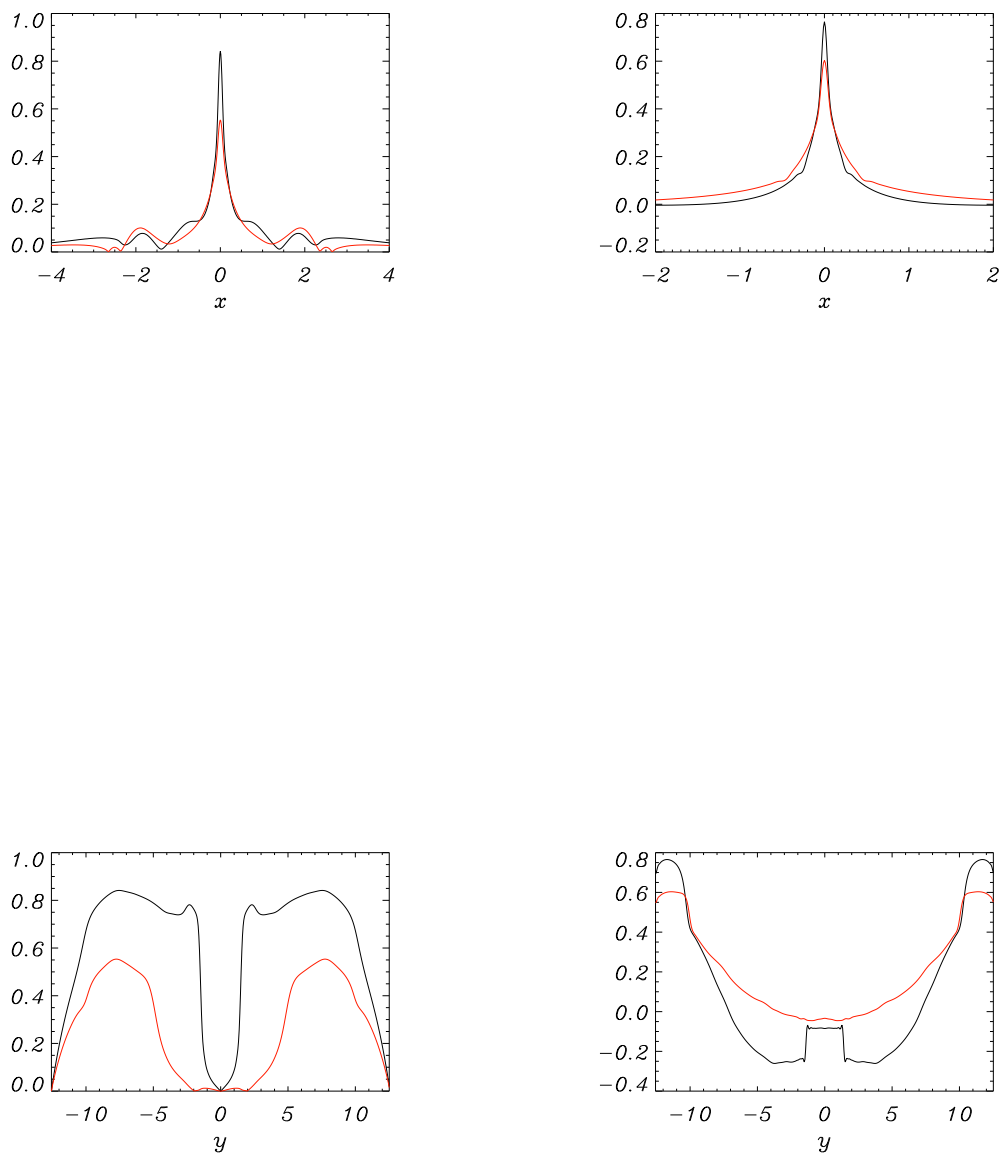

Figure 7: Profiles on the $z=0$ section of $v_{\perp}$ and $v$ as function of $x$ (top left and right frame respectively) at the $y$ coordinate corresponding to their maximum, and as function of $y$ (bottom left and right frame respectively) at $x=0$. The curves corresponding to the $c_{\beta}=0.8$ case are drawn in black, while the curves corresponding to the $c_{\beta}=0.4$ case are drawn in red. 

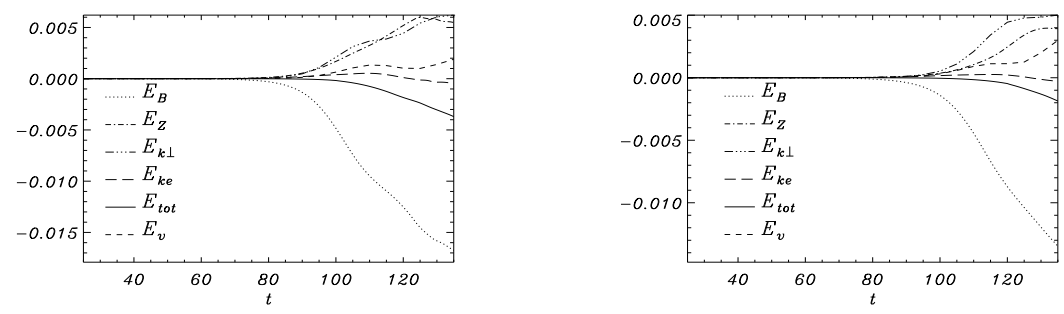

Figure 8: Normalized deviations, of the different energy contributions from their value at $t=0$, as a function of time for two different simulations. On the left is the plot corresponding to the set of parameters $c_{\beta}=0.4, d_{i}=2.4$, while on the right is the plot corresponding to the case $c_{\beta}=0.8, d_{i}=1.2 . \quad E_{V}$ refers to the parallel plasma kinetic energy $(1 / 2) \int d^{3} x v^{2}, E_{Z}$ to the parallel magnetic energy $(1 / 2) \int d^{3} x Z^{2}, E_{B}$ to the perpendicular magnetic energy $(1 / 2) \int d^{3} x\left|\nabla_{\perp} \psi\right|^{2}, E_{k e}$ to the kinetic energy associated to the parallel current density $\left(d_{e}^{2} / 2\right) \int d^{3} x J^{2}, E_{\text {kperp }}$ to the perpendicular kinetic energy $(1 / 2) \int d^{3} x\left|\nabla_{\perp} \phi\right|^{2}$ and $E_{\text {tot }}$ to the total energy $H$. 